$\begin{array}{cl}\begin{array}{cl}\text { Revue } \\ \text { de /histoire } \\ \text { des religions }\end{array} & \text { Revue de l'histoire des religions } \\ & 2 \text { Religion, secret et autorité }\end{array}$

Secrets de clôture : reliques et production de puissance sacrale (France et Italie contemporaines)

Enclosure and its Secrets: Relics and the Production of Sacral Power in Today's France and Italy

Francesca Sbardella

URL : http://journals.openedition.org/rhr/7776

DOI : 10.4000/rhr.7776

ISSN : 2105-2573

Éditeur

Armand Colin

Édition imprimée

Date de publication : 1 juin 2011

Pagination : 289-305

ISBN : 978-2200-92720-2

ISSN : 0035-1423

Référence électronique

Francesca Sbardella, «Secrets de clôture : reliques et production de puissance sacrale (France et Italie contemporaines) », Revue de l'histoire des religions [En ligne], 2 | 2011, mis en ligne le 01 juin 2015, consulté le 01 mai 2019. URL : http://journals.openedition.org/rhr/7776 ; DOI : 10.4000/rhr.7776 


\section{Secrets de clôture : reliques et production de puissance sacrale (France et Italie contemporaines)}

Sur la base d'une recherche ethnographique de terrain menée en France et en Italie, cette contribution interroge le concept de secret inhérent aux pratiques de production et de re-production de l'objet-relique dans la cloture chrétienne contemporaine. Elle s'intéresse aux traitements matériels et symboliques opérés principalement par des Carmélites sur les restes d'un saint ou d'un bienheureux. Plus encore, elle s'attache au rôle du secret dans le contrôle d'un processus et à l'apparition de compétences hiérarchisées, inscrites dans un système de communication réservé à une minorité. Il s'agit d'observer comment ces religieuses, s'étant soustraites à l'influence du clergé séculier, ont su-par le biais de pratiques dissimulées - s'aménager des niches de compétence et d'autonomie.

\section{Enclosure and its Secrets : Relics and the Production of Sacral Power in Today's France and Italy}

On the basis of ethnographic fieldwork carried out in an ItalianFrench context, the notion of secrecy is considered within production, re-production and signification practices applied to relic-objects in the contemporary Christian monastic tradition. This study looks at the material and symbolic procedures performed on the remains of a saint or a blessed, principally by Carmelite nuns. It also looks at the role of secrecy in controlling a process and at the establishment of diversified roles, hierarchical sets of skills and a confidential communication system. Having eluded the influence of the secular clergy, these nuns are women who have attained precise areas of competence and autonomy. 
Le non-dit (qui, comme le rappelle Georg Simmel, s'applique aussi au mensonge non découvert ${ }^{1}$ ) est un moyen d'asseoir une supériorité sociale, politique ou spirituelle. Le silence accompagne notre vécu quotidien; c'est la dimension dans laquelle nous prononçons nos discours, mettons en place nos comportements et instaurons nos relations avec autrui. Peut-être ne l'exprimons-nous pas, peut-être n'en avons-nous qu'une conscience limitée; il demeure que chacun de nous utilise et gère le silence pour construire des jeux de pouvoir. Le silence entre alors dans le cadre d'une volonté, d'une action intentionnelle et finalisée. Le contrôler signifie contrôler non seulement le discours, au sens technique, mais aussi la succession des actions et ce qui nous intéresse plus particulièrement ici - leur performativité.

Certains processus de construction du secret de groupe relèvent d'une démarche à la fois d'appartenance et d'exclusion. Dans le cadre d'un milieu religieux chrétien, nous nous intéresserons à l'un des secrets les plus caractérisants de la vie claustrale féminine : le travail effectué sur des restes humains, osseux et textiles, pour la production de reliques. Il s'agira de décortiquer et de déchiffrer une procédure que les actrices sociales tiennent cachée, en nous penchant d'une part sur le contenu de ce secret et, de l'autre, sur la modalité par laquelle il prend forme, se met en place et définit les rôles à l'intérieur et à l'extérieur du monastère. Nous observerons que, d'une certaine façon, ce sont le cadre et l'élaboration mêmes du secret qui font le secret. Notre recherche nous a permis d'examiner à la loupe un localisme qui, pour être clos et néanmoins intrusif, se confronte à des univers cognitifs et à des contextes culturels diffus, même en milieu laïque, et partout influents.

\section{COMMENT LE « RESTE » DEVIENT RELIQUE}

Dans le monde chrétien, les restes de personnages considérés hors du commun (qu'il s'agisse de morceaux de tissu ayant été en

1. Georg Simmel, «Il segreto e la società segreta », in Id., Sociologia, Milano, Comunità, 1998, p. 291-345 [titre original: " Das Geheimnis und die geheime Gesellschaft », in Id., Soziologie. Untersuchungen über die Formen der Vergesellschaftung, Berlin, Duncker \& Humblot, 1983, p. 257-304]. 
contact avec le corps de la personne ou de parties osseuses de ce même corps) - communément appelés reliques - sont des objets particuliers que les croyants définissent comme sacrés et auxquels est reconnue une force prodigieuse et extrahumaine qui, canalisée et utilisée selon des modalités précises que nous examinerons, est en mesure d'intervenir sur la vie humaine ou, plus simplement, sur le quotidien. Michel Albaric parle d'une « épistémologie de la chose matérielle religieuse $»^{2}$, reconnaissant ainsi la nécessité d'étudier l'histoire, la structure, la formation et les modalités d'existence sociale de ces objets.

$\mathrm{Si}$, dans le milieu ecclésiastique et pour les acteurs sociaux chrétiens, les restes sont d'emblée considérés comme des reliques, ils ne sont pas, en fait, utilisés tels quels dans des pratiques dévotionnelles. Avant cela, ils doivent être convenablement travaillés, c'est-à-dire transformés en objets plus élaborés. Il convient donc, au plan méthodologique, de faire la distinction entre objet-reste et objet-relique. Ce dernier se présente comme un objet composite, résultant de l'intégration ordonnée d'éléments hétérogènes (restes, reliquaires, accessoires). Pour en saisir pleinement la valeur, il est nécessaire de l'encadrer à l'intérieur d'un processus de transformation, par le biais duquel un simple matériau corporel devient un instrument ou un objet symbolisé sophistiqué, doté de caractères signifiants en termes religieux et dévotionnels. La distinction qui se crée entre les deux types d'objet, les objets non traités et les objets traités, est digne d'intérêt. Les premiers appartiennent à la catégorie particulière d'objets que Gian Paolo Gri appelle les « inachevés » ${ }^{3}$. Ils représentent le degré le plus bas du processus d'attribution de sens et acquièrent leur pleine identité seulement au moment où ils sont traités.

Sur la base d'une recherche de terrain ethnographique sur les objets dévotionnels en milieu claustral carmélitain et augustinien, menée en France et en Italie ${ }^{4}$, nous étudierons en détail, dans une

2. Michel Albaric, «Les objets religieux domestiques », in Encyclopédie des religions, Paris, Universalis, 1991, p. 314-315.

3. Gian Paolo Gri, «Salire verso la grazia. Strutture simboliche marginali dell'itinerario religioso », in Accademia Udinese di Scienze Lettere ed Arti (dir.), Santuari Alpini. Luoghi e itinerari religiosi nella montagna friulana. Atti del Convegno di Studio, Udine 1997, Tavagnacco (UD), Arti Grafiche Friulane, 1997, p. 76.

4. Francesca Sbardella, Antropologia delle reliquie. Un caso storico, Brescia, Morcelliana, 2007. 
optique anthropologique, l'aspect strictement matériel des pratiques mises en œuvre sur des restes reconnus comme spéciaux, d'ordinaire négligées par l'historiographie ${ }^{5}$.

À première vue, la production et la re-production de l'objetrelique se réalisent in primis sur le plan matériel. Ces opérations manuelles, accomplies par des sœurs cloîtrées spécialisées, constituent l'un des moments clé d'un long parcours visant à donner une forme et un sens au reste en question, qui sera ensuite exposé et utilisé dans le cadre de pratiques dévotionnelles et rituelles.

Le traitement des restes (textiles ou osseux) consiste à les nettoyer, à les morceler et à les disposer, avec des décorations graphiques ou des éléments en papier, sur différents types de supports (notamment des cartons ou des coussins) dans des vitrines ou des reliquaires. Bien qu'admise par le monde ecclésiastique, la manipulation de ces objets exige l'intervention de personnes qualifiées, à savoir les religieuses, précisément, qui peuvent être considérées comme des figures de médiation avec l'extrahumain.

Comment ce travail s'articule-t-il? Pour simplifier, divisons-le en macro-séquences, la première étape étant la préparation même du plan de travail. La religieuse étale une pièce de tissu sur la table et y dépose le fragment (osseux ou textile), la vitrine et les outils de travail : ciseaux, pinces coupantes, pincettes, fil et aiguille, colle, une petite casserole avec de la cire à cacheter, le tampon du monastère. L'acte consistant à étaler le drap sur la table, que la sœur accomplit avec le plus grand soin, est considéré comme indispensable à la réussite du travail et acquiert une forte valeur symbolique. D'une part, il évite le contact direct des restes avec le monde environnant; de l'autre, il permet de récupérer les minuscules morceaux résultant éventuellement de la parcellisation. À la fin du travail, les religieuses secouent le drap au-dessus de la boîte des restes ou dans un coin de la boîte contenant les outils afin que ces matériaux, qui ont à leurs yeux une valeur exceptionnelle, n'entrent pas en contact avec des objets d'usage quotidien et qu'ils ne soient pas perdus. Si,

5. Le travail de terrain s'est déroulé principalement dans des couvents carmélitains et augustiniens, deux ordres féminins qui se consacrent souvent au traitement de restes. Compte tenu de la délicatesse du sujet, j'ai choisi, afin de préserver l'anonymat des religieuses avec lesquelles j'ai collaboré et vécu pendant de courtes périodes, de ne pas révéler les noms des différents couvents, qui n'apporteraient rien à l'analyse théorique ici proposée. 
par erreur, une particule de fragment tombe par terre, les religieuses réagissent par des gestes précis : elles la ramassent, la baisent et la remettent à sa place. Chaque objet utilisé par les religieuses se rattache à tout un ensemble de signes et de signifiés. Ces actes de protection sont finalisés à la création d'un espace symbolique ordonné, isolé, séparé le plus possible du monde sensible.

Le motif de cette séparation du monde environnant revêt un certain intérêt : pour les religieuses, le contact avec des objets d'usage quotidien produit une diminution ou un appauvrissement qualitatif du pouvoir extrahumain censé résider dans le reste. Mary Douglas parle de concept de « contamination », qui permet de comprendre les limites constituées pour maintenir séparées ce que l'auteur appelle les sphères du pur et de l'impur, déjà envisagées dans la dichotomie établie par Durkheim entre sacré et profane. L'anthropologue britannique remarque que ces deux catégories constituent une modalité de contrôle sur les actions des individus et organisent aussi l'ordre social dans son ensemble ${ }^{6}$.

Après la préparation de la table de travail vient celle de la vitrine (ou du reliquaire), que l'on ouvre et à laquelle on applique la plaque portant le nom du saint ou du bienheureux, ainsi que les décorations. Généralement, le fragment est posé sur un support de papier ou de carton, découpé selon une forme géométrique ou florale et lui-même collé sur le fond. Dans les grands reliquaires, les restes reposent le plus souvent sur des coussins ou des étoffes brodés. La préparation et la décoration de la vitrine sont elles aussi considérées comme des opérations délicates. Pour les religieuses, c'est là une manière non seulement de reconnaître l'unicité du reste, mais aussi de le valoriser de la manière la plus appropriée. Le travail étant effectué pour des destinataires précis (ecclésiastiques, ordres religieux, laïcs), les sœurs tiennent compte des préférences d'un public et des exigences typiques du marché en matière d'esthétique, de fonctionnalité et de forme des objets réalisés.

Les paroles de prière marquent le début du traitement proprement dit. L'invocation d'ouverture (généralement des formules préétablies) est suivie du rosaire, récité à haute voix, ou d'une médi-

6. Mary Douglas, Purezza e pericolo. Un'analisi dei concetti di contaminazione e tabù, Bologna, il Mulino, 1993, p. 85 [Titre original : Purity and Danger. An Analysis of Concepts of Pollution and Taboo, Harmondsworth, Penguin Books, 1966]. 
tation silencieuse, le silence étant considéré ici comme une prière intérieure. La religieuse peut maintenant saisir le reste et, s'aidant des pincettes et des ciseaux, en découpe un fragment aux dimensions voulues; s'il s'agit d'un reste osseux, elle utilisera les pinces coupantes. C'est à ce moment-là que se réalisent la proximité et le contact plus ou moins prolongé avec ce qui est reconnu comme support matériel de puissance extrahumaine - une situation dont il est plus facile d'appréhender l'exceptionnalité si l'on parvient à se glisser dans la même perspective. La religieuse peut alors décider de couper le fragment en plusieurs parties, de créer de nombreux autres petits objets à vénérer. Après quoi, elle applique de la colle sur le fragment et fixe celui-ci sur le support décoratif posé sur le fond, puis remet ce dernier dans la vitrine, le fixe en passant du fil à coudre dans des trous aménagés à cet effet sur les côtés et applique le sceau de cire : la vitrine est maintenant définitivement fermée. La religieuse range les outils et nettoie la table de travail pour récupérer de menus morceaux de restes qui, éventuellement, s'y trouveraient.

Ce sont là des actes fortement standardisés, qui répondent à de petits gestes toujours accomplis d'une certaine manière et selon une séquence donnée. Les religieuses n'agissent pas sur la base de choix personnels variables, mais respectent des règles précises, une tradition conventuelle codifiée. Ajoutons que, d'un couvent à l'autre, même appartenant à des ordres différents, la procédure est assez uniforme. S'il peut apparaître des variations liées aux traditions locales et aux directives de chaque prieure, la démarche demeure fondamentalement la même. Le travail des restes renvoie à un savoir des mains, à des techniques artisanales précises qui font partie intégrante de la vie du couvent et qui caractérisent les différents rôles des religieuses. Yvonne Verdier fait observer que la gestualité des savoirs techniques possède «sa stratigraphie et son historicité propre» et s'inscrit au sein d'un ordre normatif dicté par l'usage, perçu et reconnu comme local ${ }^{7}$. Il s'agit de « façons de faire ${ }^{8}$ " précises, souvent différenciées selon le sexe : mouvements, manœuvres particulières, capacité de mettre en place des

7. Yvonne Verdier, Façons de dire, façons de faire. La laveuse, la couturière, la cuisinière, Paris, Gallimard, 1979, p. 81.

8. Ibid., p. 12. 
séries d'opérations et de stratagèmes ${ }^{9}$. Par ses modalités de transmission et d'apprentissage, le traitement des restes peut s'assimiler aux technologies artisanales, la seule différence étant qu'il est tenu secret, souvent entouré d'un halo de mystère.

Nous avons signalé que les religieuses utilisent des outils d'usage quotidien. Si elles s'efforcent, pendant la phase préparatoire, de les laisser le plus possible à l'écart de la table de travail et d'éviter tout contact avec eux, ce sont néanmoins ces objets qu'elles utilisent pendant la phase effective du traitement des restes. Conscientes de cette évidente contradiction, elles cherchent à l'annuler en usant du cadre symbolique mis en place, nous l'avons vu, au début des opérations, lorsque, avec le plus grand soin, elles préparent la table de travail. Le traitement des restes est considéré comme une activité très délicate et les religieuses avancent généralement deux motifs pour expliquer les soins particuliers qu'il exige. En premier lieu, pendant le travail, il semblerait se produire un contact avec la potentia de l'objet, c'est-à-dire avec la force, concrète et efficace, qu'il renferme ${ }^{10}$. Si cela est perçu comme un grand privilège, ce peut néanmoins être aussi source de danger. En second lieu, au moment où le reste est découpé, divisé, cette potentia pourrait être elle-même susceptible de se parcelliser.

Au plan émique, travailler les restes signifie pouvoir accéder, à travers les sens, à la force ultra-terrestre et bénéfique qu'ils renferment et pouvoir l'utiliser en ce monde, en l'orientant vers des situations, personnes ou objets spécifiques. Cela implique un contact (par l'intermédiaire, certes, de la matérialité de l'étoffe ou de la substance osseuse) avec la potentia elle-même. Il s'agit là d'une condition de proximité, de rapprochement spirituel, réalisée au travers de la matérialité. Comme le remarque Alphonse Dupront, les sens (notamment le toucher, l'odorat et la vue) jouent un rôle central dans la communication avec l'objet reconnu comme sacré $^{11}$. Pouvoir le manipuler librement est considéré comme une

9. Franco Lai, «Trasmissione e innovazione dei saperi locali», in Id., Fare e saper fare. I saperi locali in una prospettiva antropologica, Cagliari, CUEC, 2004, p. 21.

10. Marino Niola, Sui palchi delle stelle. Napoli, il sacro, la scena, Roma, Meltemi, 1995, p. 55.

11. Alphonse Dupront, Du sacré : croisades et pèlerinages, images et langages, Paris, Gallimard, 1987, p. 452. 
opportunité unique, qui n'est pas accordée à tout le monde et qui souvent produit une situation émotionnelle intense ${ }^{12}$. C'est en effet le seul moment où les religieuses peuvent voir les restes de près, les toucher, sans aucune médiation. Elles ont souvent mentionné l'exceptionnalité de ces actions. Toutefois, cette sensation de joie s'accompagne parfois d'un sentiment de crainte. Les sœurs ont le sentiment de se trouver face à une puissance mystérieuse, invisible, divine, que les seules forces humaines ne peuvent contrôler; pour certaines qu'elles soient de son existence, elles ne peuvent la voir concrètement. Elles estiment que les opérations peuvent se révéler dangereuses. D'où l'attention accordée à chaque mouvement et la nécessité de se cantonner à des gestes fortement formalisés, structurés. On retrouve là l'hypothèse d'Alphonse Dupront qui suggère que, dès lors que l'on a à faire à des objets particuliers, le toucher, en tant que moyen privilégié de "communication immédiate ${ }^{13}$ ", doit pouvoir être contrôlé par des gestes précis et temporellement circonscrits. Ainsi que le rappelle Émile Durkheim, les gestes, les langages, les attitudes qu'utilise l'individu dans la relation avec les objets considérés comme sacrés reflètent toujours l'intention de réaliser un comportement unique, étranger au plan coutumier du quotidien $^{14}$.

Si le contact avec le reste et sa manipulation sont déjà considérés comme chargés de dangerosité, il est aisé d'imaginer ce que représente l'acte de la parcellisation. Il s'agit indéniablement du moment central du traitement, dans la mesure où, pense-t-on souvent dans la tradition chrétienne, il permet d'intervenir sur la puissance même de l'objet, de la canaliser et de la rendre utilisable sur plusieurs supports. D'après les religieuses, chaque parcelle conserve la même virtus - au sens de qualité et caractère - que le reste tout entier, tout comme d'ailleurs ce dernier conserve celle du corps tout entier. L'idée qu'il n'est pas nécessaire que le corps soit intact pour que l'on puisse utiliser sa puissance est présente en théologie, note l'his-

12. Marlene Albert-Llorca, « La Vierge mise à nu par ses chambrières », Clio. Histoire, Femmes et Société, 2/1995, p. 207.

13. A. Dupront, Du sacré : croisades et pèlerinages, images et langages, op. cit., p. 452.

14. Émile Durkheim, Les Formes élémentaires de la vie religieuse, Paris, Librairie Générale Française, 1991, p. 538. 
torien Michel Kaplan, depuis le $\mathrm{v}^{\mathrm{e}}$ siècle ${ }^{15}$. Selon cette façon de voir, couper un reste en fragments n'implique pas que l'on fragmente aussi la puissance qu'il contient. Celle-ci demeure intacte dans chaque partie, même minuscule. À cet égard, Durkheim rappelle à plusieurs reprises que la puissance extrahumaine est perçue comme une essence immatérielle et fugace, qui ne se laisse pas emprisonner dans la forme de l'objet. C'est une présence représentée dans l'objet, mais qui en est indépendante et ne peut être modelée par le biais de celui-ci ${ }^{16}$.

Comme on peut l'imaginer, le traitement des restes osseux s'avère extrêmement délicat et problématique. Considérant qu'il s'agit du corps même du saint ou du bienheureux, les religieuses semblent éprouver, au moment de la manipulation, une participation émotionnelle plus intense. Ce qui crée ce trouble est la modalité même de la parcellisation. Dans un premier temps, la portion osseuse, étant un matériau dur, est découpée au moyen d'une petite scie métallique. Les morceaux ainsi obtenus sont ensuite fractionnés au moyen de pinces coupantes ou réduits en poudre dans un mortier. Ce sont là des actions singulières, que d'aucuns pourraient considérer comme de mauvais goût. En effet, non seulement on utilise un outil d'usage commun, comme pour les restes textiles, mais on fait également un usage impropre de cet outil, en l'utilisant contre un corps et non contre une chose.

Rappelons qu'il s'agit d'opérations que les autorités ecclésiastiques n'acceptent et ne reconnaissent pas pleinement. À cet égard, on a vu alterner, dans la pensée ecclésiastique, des moments de ferme condamnation (surtout du $\mathrm{III}^{\mathrm{e}}$ au $\mathrm{VII}^{\mathrm{e}}$ siècle) et des périodes plus tolérantes, comme par exemple à la fin du Moyen Âge et au début de l'Âge classique (principalement entre le XIII ${ }^{\mathrm{e}}$ et le XVI ${ }^{\mathrm{e}}$ siècles) ${ }^{17}$. Dans les textes canoniques actuels, les références - déjà extrêmement rares - relatives à la fragmentation de restes tendent à condamner avec force celle concernant des parties du corps. Toutefois, on

15. Michel Kaplan, « De la dépouille à la relique : formation du culte des saints à Byzance du $\mathrm{V}^{\mathrm{e}}$ au XII ${ }^{\mathrm{e}}$ siècle », in Edina Bozóky, Anne-Marie Helvétius (éd.), Les Reliques. Objets cultes, symboles, Turnhout, Brepols, 1999, p. 24.

16. É. Durkheim, Les Formes élémentaires de la vie religieuse, op. cit., p. $539-540$.

17. Luigi Canetti, «Culto dei santi e dissezione dei morti tra antichità e medioevo », Rivista di storia e di letteratura religiosa, 35-2/1999, p. 241-278. 
ne trouve pas de commentaires approfondis justifiant cette position. Le Concile œcuménique Vatican II marque un intérêt décroissant de l'Église catholique envers toutes les problématiques liées au culte des reliques ${ }^{18}$. Le texte qui fournit le plus d'indications sur la pratique de la fragmentation des corps morts est probablement le récent Directoire sur la piété populaire et la liturgie ${ }^{19}$, publié par la Congrégation pour le Culte Divin et la Discipline des Sacrements. Il s'agit d'un texte normatif destiné aux évêques, contenant des principes et des orientations relatifs à la liturgie. Mais de nombreux couvents choisissent de l'ignorer et la pratique de fragmenter des parties osseuses est donc communément répandue.

\section{Un SAVOIR CACHÉ}

L'acte de traiter les restes, quels qu'ils soient, est perçu par les religieuses (même par celles qui ne le font pas et par celles qui n'appartiennent pas à la clôture) comme un acte différent, autre, qui ne relève pas du simple travail manuel :

Quand elles [= les sœurs qui confectionnent les reliques] font le geste de couper, c'est un geste liturgique parce qu'elles le font pour constituer un objet important ou sacré. [...] c'est quelque chose qui exprime une intention du cœur et de l'esprit, une intention levée vers le Seigneur ${ }^{20}$.

Définir le traitement des reliques comme un « geste liturgique » signifie l'inscrire dans le culte tel qu'il est fixé par la tradition communautaire et par l'autorité ecclésiastique. Dans la tradition chrétienne, l'acte liturgique est l'action religieuse (cérémonielle ou rituelle) par laquelle une communauté professe sa foi et rend, sous une forme légitime et parfois largement confirmée par la coutume, le culte à la divinité. La vie même des religieuses cloîtrées est rythmée par un acte liturgique de base : la « liturgie des

18. Nicole Herrmann-Mascard, Les Reliques des saints. Formation coutumière d'un droit, Paris, Klincksieck, 1975; Franco Magnani, «Le reliquie : tra l'oblio della teologia e il rinnovato interesse della cultura post-moderna », Liturgia, $173 / 2002$, p. 506-527.

19. Congregazione per il Culto Divino e la Disciplina dei Sacramenti, Direttorio su pietà popolare e liturgia. Princìpi e orientamenti, Città del Vaticano, Libreria Editrice Vaticana, 2002.

20. Déclaration de sœur P., France, 11 août 2002 (Je souligne). 
heures », qui articule les différents moments de prière de la journée. Considérer le traitement des reliques comme un acte liturgique signifie donc le faire rentrer dans les moments de prière communautaires et non seulement dans ceux consacrés au travail manuel courant (ouvrages de broderie et de couture, préparation de gâteaux, production d'objets en céramique). Même lorsqu'elles ne se réfèrent pas explicitement à l'acte liturgique, les sœurs - et plus particulièrement les religieuses italiennes - ne parlent pas moins de « prière ".

Cette volonté de préciser la nature du travail effectué est soulignée par des choix lexicaux précis. Pour elles-mêmes, les religieuses n'utilisent jamais les termes lavoro et travail, et reprennent immédiatement l'interlocuteur externe qui les utilise - cette imprécision étant même perçue comme un manque de respect à l'égard de leur activité. Traiter les restes, donc, est considéré comme un moment d'intime dévotion qui entre dans les aspects de la vie contemplative. Les religieuses semblent éprouver un certain malaise spirituel et culturel vis-à-vis de cette activité et ressentir le besoin de la requalifier positivement. Elles la traduisent alors dans le code religieux typique de la clôture. Ainsi conceptualisent-elles en prière le travail manuel effectué sur les reliques. Les termes utilisés mettent en évidence l'aspect dévotionnel et cultuel de l'acte en question. Les sœurs tendent à faire passer au second plan l'idée de l'activité matérielle liée à la production d'un bien ou, quoi qu'il en soit, à l'obtention d'un produit d'utilité individuelle ou sociale. Les pratiques corporelles et spirituelles semblent se superposer. L'aspiration à l'élément mystique, typique des milieux cloîtrés, est favorisée par des identités corporelles précises. D'après Marcel Mauss $^{21}$ - repris ensuite par Pierre Bourdieu ${ }^{22}$-, c'est grâce aux mouvements et aux pratiques techniques acquises par l'individu en tant que membre d'une société que l'on peut intervenir sur l'émotion et sur l'inconscient. Selon lui, tous les états mystiques ont à la base des techniques précises du corps.

Il convient de souligner une contradiction évidente. Quand les religieuses racontent aux autres (laïcs ou ecclésiastiques) leur acti-

21. Marcel Mauss, "Les techniques du corps», Journal de psychologie XXXII, 3-4/1936, p. 45.

22. Pierre Bourdieu, Ce que parler veut dire. L'économie des échanges linguistiques, Paris, Fayard, 1982. 
vité, elles cherchent, nous l'avons dit, à la présenter comme un acte dévotionnel. Par contre, quand elles en parlent entre elles, elles utilisent des expressions fortement caractérisées au sens matériel. L'expression italienne « confezioniamo le reliquie » et l'expression française " on fait les reliques », toutes deux typiques de la langue parlée et utilisées exclusivement au sein du milieu claustral, laisseraient supposer que les religieuses elles-mêmes ne considèrent pas tous les restes comme pouvant en tant que tels être des reliques: en d'autres termes, pour devenir relique, le reste doit être traité ou confectionné selon des modalités précises qui relèvent avant tout d'un savoir-faire. Hors du monde conventuel, en revanche, les religieuses semblent vouloir se représenter par le biais de fonctions et de rôles exclusivement liés au milieu contemplatif et dévotionnel, et non à un contexte pratico-manuel.

N'oublions pas que nous nous trouvons dans un milieu fortement séparé du monde laïque - au plan spatial, certes, du fait de la « clôture » elle-même, mais aussi et surtout par effet du silence qui entoure toutes les opérations liées au traitement des restes : une barrière dont la pleine signification ne s'exprime pas tant vis-à-vis des laïcs (qui, dans la plupart des cas, ignorent les opérations mises en place pour produire les reliques et, d'ailleurs, ne s'interrogent que rarement à propos du produit fini) que vis-à-vis des autres religieux et religieuses. Ces derniers connaissent certainement plus en détail le processus de traitement, mais ne sont pas admis à y participer directement ou à y assister. Ainsi vient à se créer une condition privilégiée pour quelques rares sœurs, les seules qui puissent toucher et manipuler librement les restes de défunts prestigieux.

D'après G. Simmel, l'exclusion fortement accentuée de tous les étrangers donne un sentiment de propriété de force correspondante $^{23}$. Ici, l'élément discriminant, qui crée la différence entre celui qui sait et celui qui ne sait pas, est le secret lié au travail de l'objet chargé de puissance. Si les laïcs n'imaginent pas que l'objet en question est soumis à des manipulations structurées et répétées, les autres religieux (extérieurs au couvent) ignorent, quant à eux, la façon dont elles se produisent. Il y a donc deux niveaux de secret : celui du non-savoir et celui consistant à ne pas savoir comment. Dans le premier cas, le sentiment qu'ont les sœurs

23. G. Simmel, «Il segreto e la società segreta », art. cité, p. 310. 
d'appartenir à un groupe privilégié leur vient du fait de partager un savoir qui leur est réservé, conscientes que les autres ne savent même pas qu'il y a quelque chose qu'ils ne savent pas. Dans le second cas, le caractère identitaire se nourrit de la conscience que les autres religieux, exclus de ce savoir, sont au courant de son existence mais ignorent comment il se pratique ou s'utilise. C'est alors qu'entre en jeu, de manière explicite et reconnue, la diversification des rôles et des compétences hiérarchisées à l'intérieur du cadre religieux. Ici, c'est l'existence même de ce savoir secret qui crée des niveaux et des pouvoirs différents, en ordonnant et différenciant le groupe social à l'intérieur de la communauté religieuse et laïque dans son ensemble. Dans les deux cas, les autres ignorent autant les pratiques réellement mises en place par les sœurs que le vocabulaire qui s'y rapporte. Notons en effet qu'il s'est créé dans les différents couvents de clôture, autour du traitement concret de l'objet, un ensemble complexe et spécifique de termes et de façons de parler, une sorte de répertoire lexical qui permet aux sœurs de se comprendre rapidement tout en maintenant les autres à l'écart. En ce sens s'est élaboré un système de communication interne, réservé aux religieuses du couvent. Toutes, même celles qui ne traitent pas personnellement les restes, sont au courant de la procédure et peuvent d'ailleurs être appelées à s'en charger. Elles se sentent autres par rapport au monde extérieur et perçoivent très fortement la sensation d'être dans une dimension spirituelle différente, même s'il n'existe pas réellement de savoir particulier que les sœurs seules possèdent.

Dans la pratique, en effet, les objets considérés comme sacrés sont soumis à un travail artisanal de type traditionnel, réalisé, en outre, à l'aide d'outils tout à fait ordinaires (colle, ciseaux, pinces coupantes, fil et aiguille). C'est peut-être là l'un des aspects les plus frappants, lorsque l'on assiste à ces opérations. Mais, précisément parce qu'elles touchent à une puissance extrahumaine, ces actions demeurent cachées. Il ne s'agit pas en soi de pratiques exceptionnelles, mais elles le deviennent dès lors que l'on tait leur existence aux laïcs et que les autres religieux, tenus à distance, peuvent seulement les imaginer. On laisse en fait supposer que le traitement du sacré, justement parce qu'autre par excellence, ne peut se faire selon des modalités usuelles ou avec des instruments empruntés à la vie quotidienne. 
Les sœurs cloîtrées laissent entendre que les restes sont traités de manière tout à fait particulière et créent un halo de mystère, qui garantit une discrétion totale. Lorsque l'on met en place une forme de savoir secret, fait remarquer Cesare Poppi, ce qui est important dans la plupart des cas, n'est pas tant le contenu de la communication que son contrôle. Ce qui constitue le secret est souvent le « cadre » même de la situation, plus que son contenu réel ${ }^{24}$.

Ici, c'est aussi la modalité de transmission restreinte qui concourt à créer le « cadre » d'isolement et de valorisation du savoir en question. Il est en effet transmis comme une compétence initiatique, entourée de réserve et de secret. L'acte de travailler les restes est considéré comme relevant d'une capacité extraordinaire, qu'il n'est pas donné à toutes les sœurs d'acquérir et qui doit être léguée avec attention. C'est un travail fait d'une multitude de petits gestes qui doivent être appris d'une certaine manière, puis accomplis avec une extrême précision.

La présence d'un contrôle, d'une direction, est aisément vérifiable, étant donné que c'est généralement la prieure du couvent qui établit que telle ou telle personne fait tel ou tel travail, dont, entre autres, la gestion des restes et des reliques. Ce choix, non impositif, tient compte des intérêts et des aptitudes personnelles des différentes religieuses, comme c'est le cas, du reste, dans toute famille. Des actes qui, nous l'avons vu, sont en soi assez simples se chargent ainsi d'une valeur additionnelle en termes de compétence et de spécialisation. Quand, pendant les interviews, les sœurs racontent le moment où le travail leur a été confié, elles soulignent la joie et la fierté qu'elles ont alors éprouvées. Dans leur optique, ce travail équivaut à être en présence des saints, à se confronter avec leur vie. La proximité physique avec leurs restes devient en premier lieu un partage d'intentions, faisant naître le désir de les prendre comme modèle.

Ainsi que nous l'avons souligné, une fois que le reste a été mis en place dans la vitrine ou dans le reliquaire, le contact direct devient impossible, y compris pour les sœurs qui s'en sont occupées. Le moment du traitement est donc le seul pendant lequel il leur est permis de toucher librement les restes. Toutefois, même si

24. Cesare Poppi, «Sigma! The Pilgrim's Progress and the Logic of Secrecy ", in Mary H. Nooter (éd.), Secrecy: African Art that Conceals and Reveals, New York-Prestel-Munich, The Museum for African Art, 1993, p. 202. 
les actes doivent être accomplis selon des modalités précises - en général à l'aide d'instruments de médiation -, la religieuse dispose d'une certaine liberté d'action qui, plus que dans les manipulations proprement dites (celles-ci étant, nous l'avons vu, standardisées), s'exprime dans la possibilité de faire durer le travail, de prolonger ne serait-ce que de quelques secondes le contact direct avec le reste. Il s'agit d'une manipulation autorisée. Les sœurs elles-mêmes le disent : elles utilisent des instruments, par exemple les pincettes, pour saisir et arranger le fragment, mais elles peuvent aussi se servir de leurs mains; parfois, même, le contact direct facilite et améliore le travail. C'est pourquoi il leur arrive de s'attarder à effleurer le reste et à l'admirer, comportement qu'elles vivent comme un acte de vénération et, en même temps, comme une demande de protection.

Au cours de l'enquête, lorsque j'ai souhaité parler des reliques et de leur production avec des représentants du clergé français et italien, il m'a souvent été répondu de m'adresser directement aux femmes, un religieux occupant une certaine position ne se préoccupant pas - comme cela a été clairement exprimé - de ce type d'activité, jugée marginale par rapport à l'engagement spirituel d'un homme d'Église. Il est néanmoins intéressant de noter que, malgré sa prise de position, le clergé finit par reconnaître le secret et par respecter les conséquences qui en découlent au plan pratique : quand les religieux souhaitent faire traiter des restes leur appartenant, c'est en effet aux moniales qu'ils s'adressent, reconnaissant par là le rôle spécialisé qu'elles se sont formé. Le secret fonctionne indépendamment de son contenu et détermine des effets concrets au plan social, même si certaines des parties en cause croient (ou affirment croire) qu'il n'a que peu d'importance ou ne présente qu'une valeur contractuelle minime. Éclairante, à cet égard, est la position de Simmel, lorsqu'il rappelle qu'il y a autour de tout homme - au sens d'individu, mais aussi de groupe - une sphère idéale que l'on ne peut pénétrer sans détruire « la valeur de personnalité de l'individu ${ }^{25}$ », en d'autres termes, sa caractérisation identitaire. Et c'est précisément autour de cette sphère idéale du secret - que nous pouvons également appeler pacte - que se construisent les dynamiques relationnelles.

25. G. Simmel, « Il segreto e la società segreta », art. cité, p. 301. 
Plus encore que par le passé, la thésaurisation des objets auxquels est attribuée une valeur sacrale, leur traitement, leur diffusion et leur utilisation sont confiés à des religieuses, pour la plupart cloîtrées. De fait, celles-ci finissent par bénéficier d'un vaste champ d'action et peuvent se servir des reliques en se prévalant d'une grande liberté. Les savoirs et les techniques qui s'y rapportent sont leur monopole. Transmis dans le milieu fermé de la tradition conventuelle, ils offrent aux religieuses une possibilité de se créer une place aux côtés des hommes d'Église. Bien que le travail des restes entre de plein droit parmi les tâches que les sœurs accomplissent au quotidien pour assurer leur subsistance (comme la couture, la fabrication des hosties), les religieuses tendent à souligner qu'il s'agit d'actions extrêmement délicates, exigeant compétence, patience et précision.

Ainsi se trace une nette division sociale du travail, fondée non seulement sur l'appartenance à la classe religieuse, mais aussi, au sein de celle-ci, sur la différence de genre. Claude Langlois parle de "dimorphisme sexuel ${ }^{26}$ ", non seulement au sens d'une diversité parmi les pratiquants laïques, par effet de laquelle il y a davantage de pratiquants femmes, mais aussi comme différenciation de rôles à l'intérieur du monde religieux lui-même ${ }^{27}$. Selon cette analyse, l'on distingue, en l'occurrence, des positions et des compétences religieuses propres à chaque catégorie sexuelle, qui s'attribue et s'aménage des spécialisations distinctives et caractérisantes où le secret joue un rôle déterminant dans la recherche et la préservation d'un monopole.

De nombreux auteurs se sont intéressés à la position féminine visà-vis du supranaturel ${ }^{28}$. Dans la culture occidentale, les femmes ne sont que partiellement intégrées dans les pratiques religieuses. Elles

26. Claude Langlois, « Toujours plus pratiquantes. La permanence du dimorphisme sexuel dans le catholicisme français contemporain », Clio. Histoire, Femmes et Société, 2/1995, p. 229.

27. Étienne Fouilloux, «Femmes et catholicisme contemporain », Clio. Histoire, Femmes et Société, 2/1995, p. 319-329.

28. Marlène Albert-Llorca, "La Vierge mise à nu par ses chambrières ", art. cité, p. 201-228; Katerina Seraïdari, «Dans l'intimité de la Vierge. Dévotions au féminin et au masculin en Grèce contemporaine », Clio. Histoire, Femmes et Société, 15/2002, p. 55-68; Alfonso Maria Di Nola, s.v. «Sacro/Profano», in Enciclopedia, Turin, Einaudi, 12/1981, p. 313-362; Agnès Fine, Claudine Leduc, « Femmes et religions », Clio. Histoire, Femmes et Société, 2/1995, p. 5-16. 
sont exclues de la plupart des actes constitutifs du culte, éloignées de la sphère sacrificielle ou reléguées dans des positions secondaires. On peut distinguer deux modalités d'accès au supranaturel : l'accès masculin, lié au sacerdoce et donc à la célébration du mystère de l'eucharistie, et l'accès féminin, qui peut se réaliser uniquement dans la divination et la prophétie. Il est clair que le premier est contrôlé et officialisé par l'institution ecclésiastique, tandis que l'autre semble se soustraire à son contrôle et être plus indépendant et direct. Avec les reliques et par le secret, les femmes semblent avoir réussi, au sein d'une institution religieuse qui les subordonne, à se construire des rôles privilégiés quant au rapport avec le sacré. Elles s'aménagent des niches de compétence spécifiques et, s'étant soustraites à l'autorité masculine, assurent ainsi une médiation privilégiée avec le divin - médiation réifiée dans le secret lui-même.

francesca.sbardella@unibo.it 